\title{
Development of High Temperature Acoustic Emission Sensing System Using Fiber Bragg Grating
}

\author{
Dandan PANG ${ }^{1,2^{*}}$, Qingmei SUI ${ }^{3}$, Ming WANG ${ }^{1,2}$, \\ Dongmei $\mathrm{GUO}^{1}$, and Yaozhang $\mathrm{SAI}^{3}$ \\ ${ }^{1}$ School of Information and Electrical Engineering, Shandong Jianzhu University, Jinan, 250101, China \\ ${ }^{2}$ Shandong Provincial Key Laboratory of Intelligent Buildings Technology, Jinan, 250101, China \\ ${ }^{3}$ School of Control Science and Engineering, Shandong University, Jinan, 250061, China \\ *Corresponding author: Dandan PANGＥ-mail: pangdan58@163.com
}

\begin{abstract}
In some applications in structural health monitoring (SHM), the acoustic emission (AE) detection technology is used in the high temperature environment. In this paper, a high-temperature-resistant AE sensing system is developed based on the fiber Bragg grating (FBG) sensor. A novel high temperature FBG AE sensor is designed with a high signal-to-noise ratio (SNR) compared with the traditional FBG AE sensor. The output responses of the designed sensors with different sensing fiber lengths also are investigated both theoretically and experimentally. Excellent $\mathrm{AE}$ detection results are obtained using the proposed FBG AE sensing system over a temperature range from $25^{\circ} \mathrm{C}$ to $200{ }^{\circ} \mathrm{C}$. The experimental results indicate that this FBG AE sensing system can well meet the application requirement in $\mathrm{AE}$ detecting areas at high temperature.
\end{abstract}

Keywords: Optical sensor; high temperature; fiber Bragg grating; acoustic emission

Citation: Dandan PANG, Qingmei SUI, Ming WANG, Dongmei GUO, and Yaozhang SAI, "Development of High Temperature Acoustic Emission Sensing System Using Fiber Bragg Grating,” Photonic Sensors, 2018, 8(1): 56-62.

\section{Introduction}

When the monitored materials are undergoing deformation, the internal cracks may generate and further extend, which will severely endanger the safety and reliability of the monitored structure. The acoustic emission (AE) monitoring technology is an excellent means for structural health monitoring (SHM) [1-3]. The AE sensors collect AE signals produced by the defects of the monitored structures. Through analyzing the feature parameters of the $\mathrm{AE}$ waveform, such as event count, amplitude, and duration, the position and severity of the AE source can be evaluated. In the situation of some SHM areas, the $\mathrm{AE}$ monitoring technique is required under the high temperature condition.

Previous studies have shown some methods for the $\mathrm{AE}$ detecting technique applied in the high temperature environment $[4,5]$. The piezoelectric transducer (PZT) used in high temperature for $\mathrm{AE}$ monitoring is usually made of high temperature piezoelectric material, which is expensive and liable to electromagnetic disturbance [6, 7]. Another method for temperature AE detecting has been reported by Sun et al. [8], Dixon et al. [9], and Cheon et al. [10]. In their studies, buffer rods were utilized to connect the common PZT sensor and the high temperature materials to be measured. However,

Received: 24 July 2017 / Revised: 22 August 2017

(C) The Author(s) 2017. This article is published with open access at Springerlink.com

DOI: $10.1007 / \mathrm{s} 13320-017-0454-9$

Article type: Regular 
the installation and shape of buffer rods directly affected the reliability of the test results.

In this paper, we design an $\mathrm{AE}$ sensing system for the high temperature environment using the fiber Bragg grating (FBG) as the sensing element. We compare the output responses of the detection in an aluminum alloy (Al-alloy) plate between a traditional FBG AE sensor and the new designed FBG AE sensor. To investigate the output characteristics of the designed sensor, the sensing length of the sensor is gradually changed. A high temperature $\mathrm{AE}$ detecting experiment is performed using the designed high-temperature-resistant $\mathrm{AE}$ sensing system over a temperature range from the ambient temperature to $200{ }^{\circ} \mathrm{C}$.

\section{High temperature FBG AE sensor fabrication}

The FBG used in this paper is written in a high temperature resistance optical fiber surrounded by an outer polymide coating. The diameter of the outer coating, the middle cladding, and the silica core are respectively $145 \mu \mathrm{m}, 125 \mu \mathrm{m}$, and $10 \mu \mathrm{m}$, as shown in Fig. 1. The range of the appropriate operating temperature of the $\mathrm{FBG}$ is from $-50{ }^{\circ} \mathrm{C}$ to $400{ }^{\circ} \mathrm{C}$.

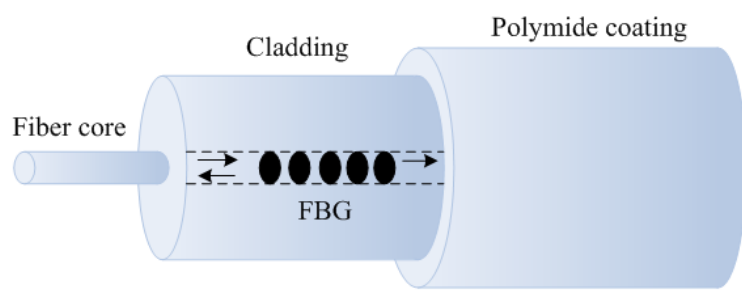

Fig. 1 Structure of the FBG written in a polymide coated optical fiber.

Figure 1 shows the principle of FBG sensing. The FBG is formed by writing a periodic modulation of the refractive index along a short section in the polymide-coated optical fiber [11]. The incoming light with the specific wavelength is reflected by the FBG based on the Bragg law:

$$
\lambda_{B}=2 \overline{n_{\text {eff }}} \Lambda
$$

where $\lambda_{B}$ is the central wavelength of the reflected spectrum of the FBG, $\Lambda$ is the grating period, and $\overline{n_{\text {eff }}}$ is the effective refractive index of the optical fiber core.

According to (1), $\lambda_{B}$ changes with the ambient conditions, such as temperature, strain, and stress. When the FBG is subjected to AE waves, $\lambda_{B}$ is evaluated as the sum of two effects: one is the geometric effect due to the modulation of the grating period under $\mathrm{AE}$ waves; the other is the photoelastic effect due to the change in refractive index [12]. In this case, (1) can be rewritten as

$$
\begin{gathered}
\lambda_{B}=2 \overline{n_{\text {eff } 0}} \Lambda_{0}\left\{1+\varepsilon_{\mathrm{AE}}\left(1-\frac{{\overline{n_{\text {eff } 0}}}_{2}^{2} .}{}\right.\right. \\
\left.\left.\left[p_{12}-v \cdot\left(p_{11}+p_{12}\right)\right]\right)\right\}
\end{gathered}
$$

where $\varepsilon_{\mathrm{AE}}$ denotes the strain field along the fiber generated by AE waves, $p_{11}$ and $p_{12}$ are the valid photoelastic coefficients, and $v$ is the ratio of Poisson.

Figure 2 shows a schematic drawing for the designed high temperature FBG AE sensor. The substrate of the designed sensor is a polymide plate, which is a good medium for AE waves. The dimensions of the substrate plate are $22 \mathrm{~mm} \times 5 \mathrm{~mm}$ with $2 \mathrm{~mm}$ thickness. This new designed FBG AE sensor has been verified to be insensitive to the mechanical strain applied on the monitored structure [13].

As shown in Fig. 2(a), the grating section of the FBG is not directly glued on the substrate plate. The optical fiber is bonded on the substrate plate using a heat-resistant adhesive at a distance of $L / 2$ from the grating section. $L$ is defined as the sensing length of the designed sensor [see Fig. 2(a)]. The end of the unbonded optical fiber is submersed in an index-matching gel to prevent noise due to back reflections. The resonance frequency $f_{n}$ of the designed sensor can be expressed as

$$
f_{n}=\frac{2 n-1}{4 L} c_{f}
$$

where $n$ is the order of the AE wave mode, and $c_{f}$ is the velocity of the AE wave along the fiber. The first theoretical resonant frequency $f_{1}$ of the designed sensor is plotted in Fig. 3(a). The first resonant 
frequency $f_{1}$ decreases as $L$ increases. The bandwidth value of the resonant peak is shown in Fig. 3(b). The bandwidth of the resonant peak decreases with an increase in $L$.

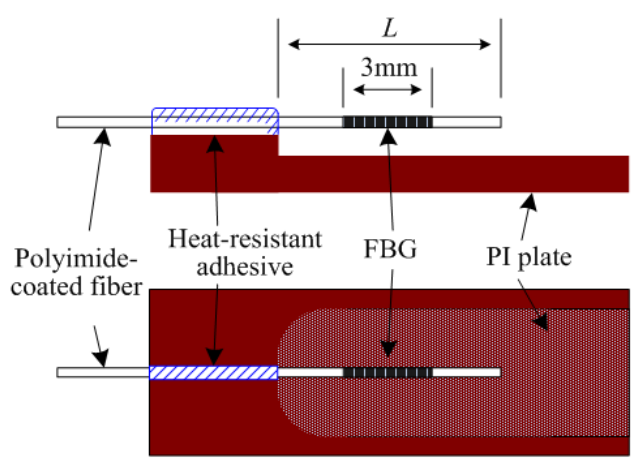

(a)

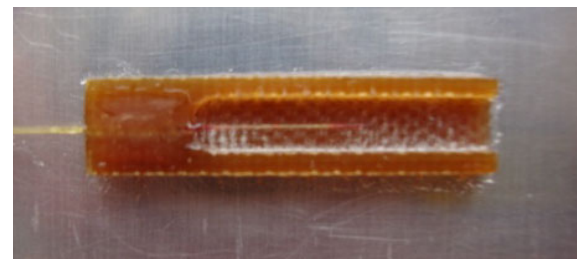

(b)

Fig. 2 Structure of the high temperature FBG AE sensor.

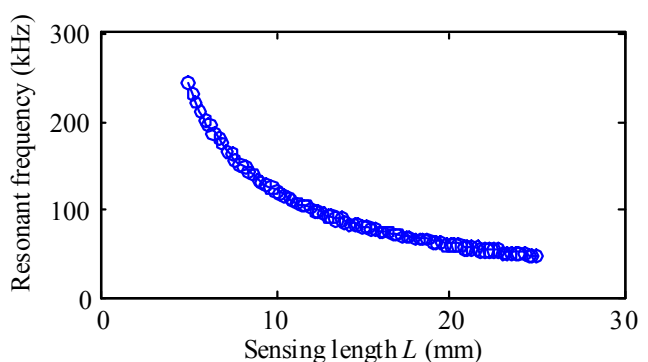

(a)

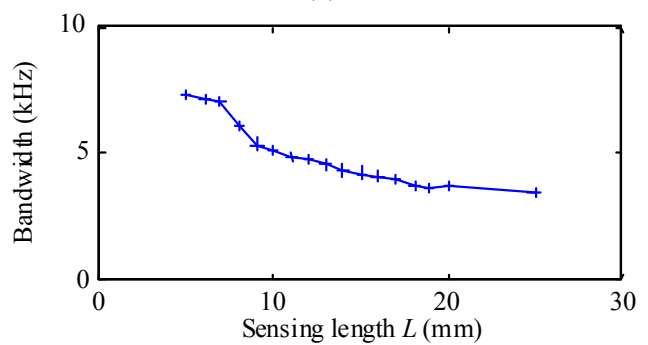

(b)

Fig. 3 Theoretical sensing characteristics of the designed sensor: (a) theoretical $f_{1}$ changes with $L$ and (b) bandwidth of resonance peaks changes with $L$.

\section{Experiments and results}

Experimental instrumentation consists of tunable laser, $\mathrm{AE}$ sensors, filters, photodetectors, amplifiers, and analysis software. The designed FBG-AE sensor and the traditional FBG-AE sensor are mounted on the surface of the Al-alloy plate as shown in Fig. 4. The dimensions of the plate are $400 \mathrm{~mm} \times 400 \mathrm{~mm} \times$ $3 \mathrm{~mm}$. The simulated AE signals are generated using a PZT actuator that is connected with a waveform generator. The PZT actuator is glued at the center of the plate.

The AE signals are detected by the FBG sensors and then through one port of the circulators $\left(\mathrm{C}_{1}\right.$ and $\mathrm{C}_{2}$ ) transmitted to the photodetectors with built-in filters. The frequencies of the built-in bandpass filters are set at $100 \mathrm{kHz}-300 \mathrm{kHz}$. The output signals of the photodetectors are amplified with a gain set at $30 \mathrm{~dB}$. The amplified $\mathrm{AE}$ signals are processed and recorded using an $\mathrm{AE}$ system.

Because of the unrepeatable characteristic of the simulated AE signals, a direct comparison test between the two different FBG AE sensors is carried out by using two sensors to detect the AE signals at the same time. The two sensors are placed parallel to each other in a line and laid on the plate at a distance of $100 \mathrm{~mm}$ from the PZT actuator, as shown in Fig. 4. The measured signals from the experiment are shown in Fig. 5.

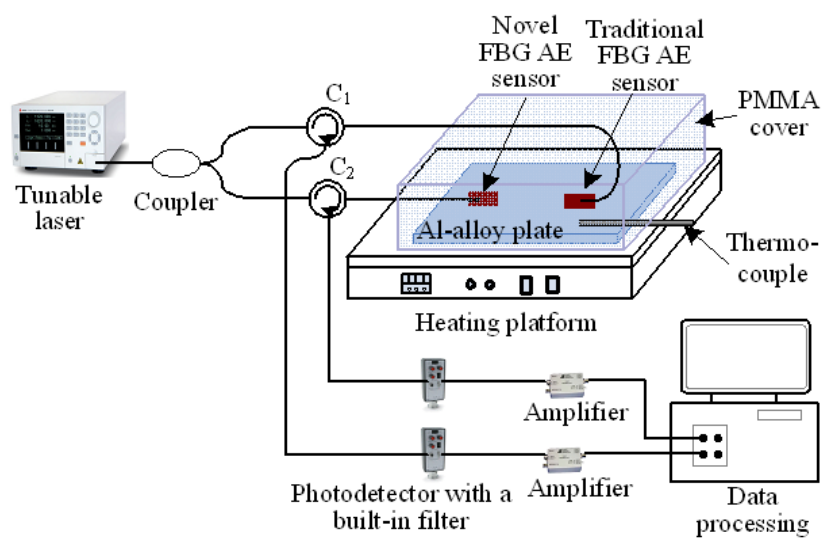

Fig. 4 Schematic of the experimental setup.

Comparing the time and frequency responses between the traditional and novel FBG AE sensors, we can see that the signal amplitude of the novel FBG AE sensor is obviously higher than that of the traditional one. Therefore, the new designed sensor 
has a good signal-to-noise ratio (SNR) for the $\mathrm{AE}$
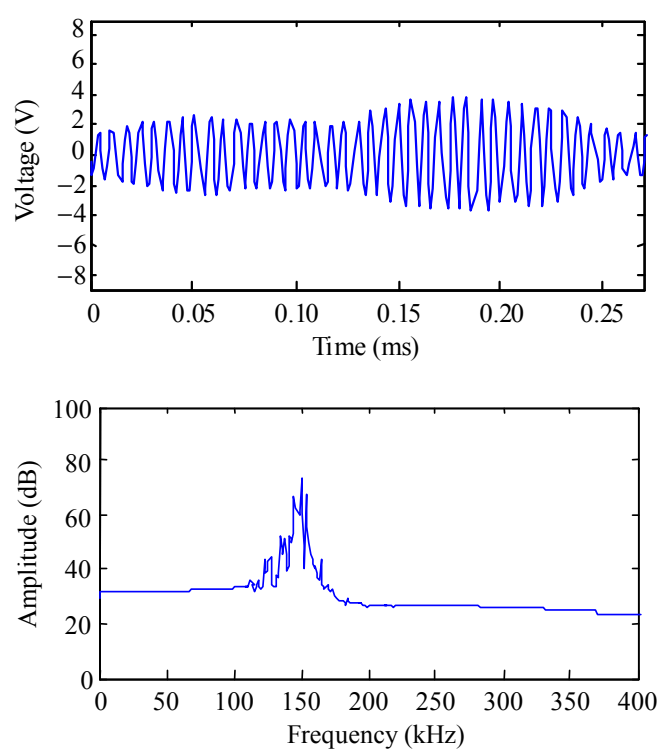

(a) wave detection.
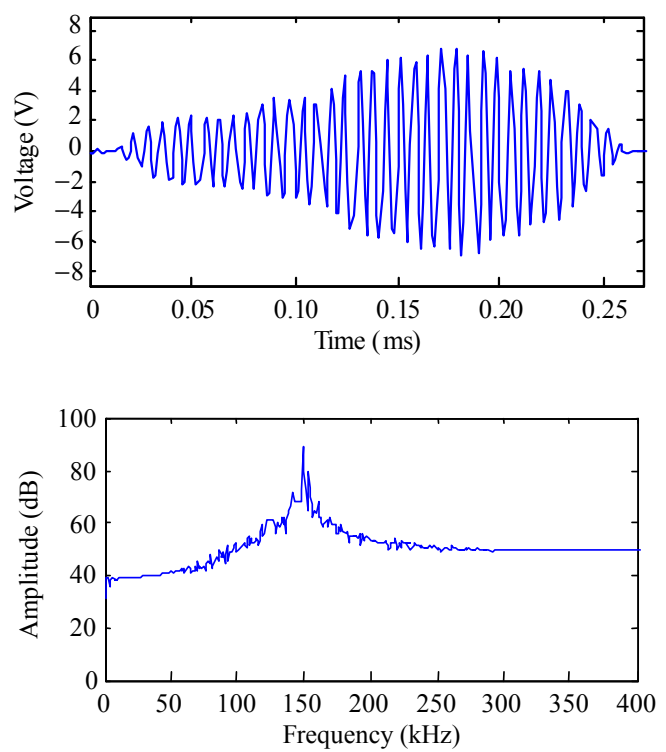

(b)

Fig. 5 Output response of traditional (a) and novel (b) FBG AE sensors.

After the experiment of comparison between the novel and traditional FBG AE sensors, we investigate the relationship between the response characteristics and the sensing length $L$ of the novel FBG AE sensor.

Between measurements, three novel FBG AE sensors are designed with different sensing lengths $L$. As mentioned in Section 2, when $L<3 \mathrm{~mm}$, the FBG itself is glued to the substrate plate. The output responses of the designed sensors are plotted in Fig. 6, with different sensing lengths $L$. According to (3) and Fig. 3, the sensing length $L$ affects the output response of the designed FBG AE sensors theoretically.

From the time response of the novel sensors, we can see that the single cycle of the collected signal becomes shorter as $L$ decreases. For every graph, three resonant frequencies of the output response of the sensors with different $L$ are obtained and listed in Table 1. The resonant frequency of the sensor shows a clear decrease with an increase in $L$. We observe that the theoretical values shown in Table 1 and Fig. 3 well coincide with the measured results.
Table 1 Resonant frequency of the output response of the sensors with different $L$.

\begin{tabular}{ccc}
\hline$L(\mathrm{~mm})$ & \multicolumn{2}{c}{ Resonant frequency of the output response $(\mathrm{kHz})$} \\
& Theoretical value & Experimental value \\
\hline 20 & 60.75 & 59.57 \\
8 & 151.875 & 149.4 \\
4 & 303.75 & 298.8 \\
\hline
\end{tabular}

In order to detect $\mathrm{AE}$ signals at high temperatures, the test apparatus shown in Fig. 7 is utilized. The Al-alloy plate is heated on a heating platform, starting at $25^{\circ} \mathrm{C}$ and increasing to $200{ }^{\circ} \mathrm{C}$. The measurement temperature is calibrated by a thermocouple. A $0.5-\mathrm{m}$-long steel rod is used as the wave guide to connect the PZT actuator with the heated plate. On one end, the PZT actuator is fixed on the steel rod, while the other end is placed on the Al-alloy plate. The PZT actuator is driven by a waveform generator to generate the simulated $\mathrm{AE}$ waves. The new designed $\mathrm{AE}$ sensor is at $10-\mathrm{mm}$ distance from the AE source. The AE sensor is mounted using a high temperature resistant silicon sealant to ensure a good coupling. The sealant has a 
maximum operational temperature of $300{ }^{\circ} \mathrm{C}$.

Figure 8 shows the frequency response obtained from high-temperature $\mathrm{AE}$ experiment at up to $200{ }^{\circ} \mathrm{C}$. The scales on all figures are the same. The
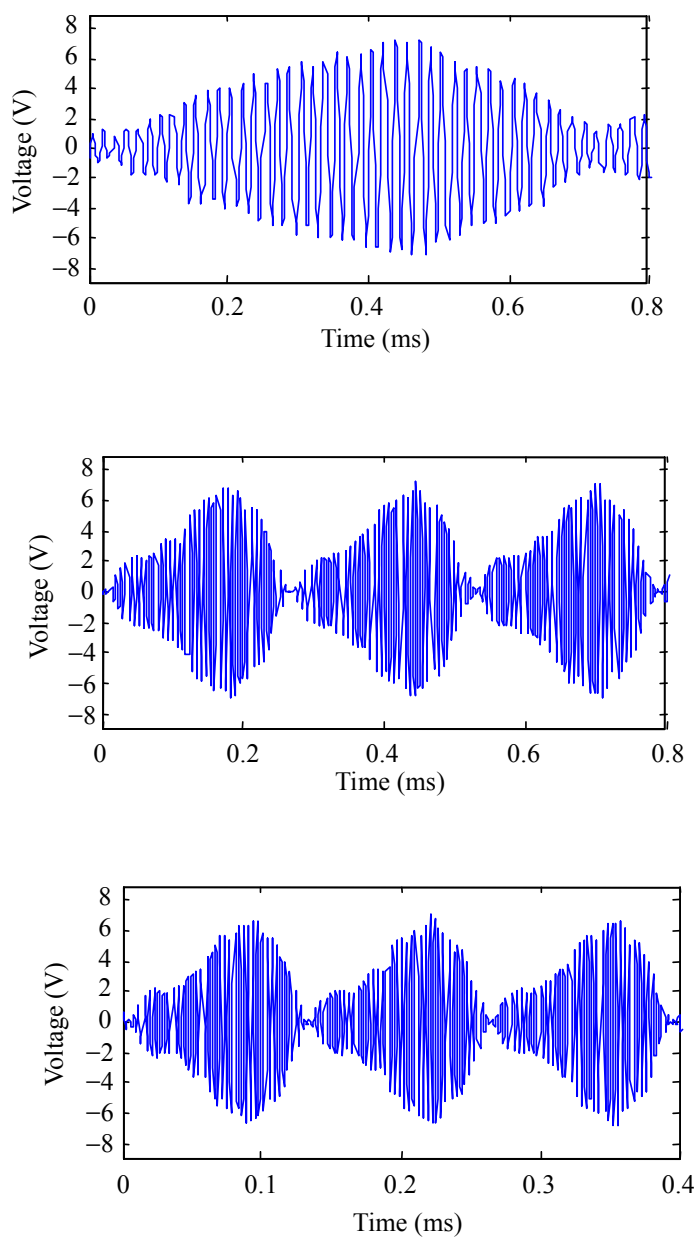

(b)
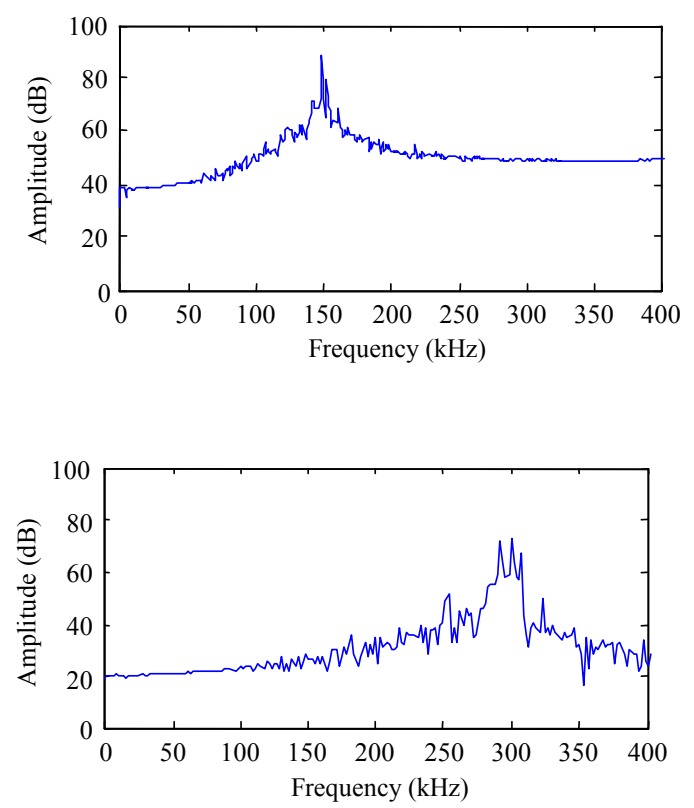

(c)

Fig. 6 Output responses of the novel FBG AE sensors with different $L$ : (a) $L=20 \mathrm{~mm}$, (b) $L=8 \mathrm{~mm}$, and (c) $L=4 \mathrm{~mm}$.

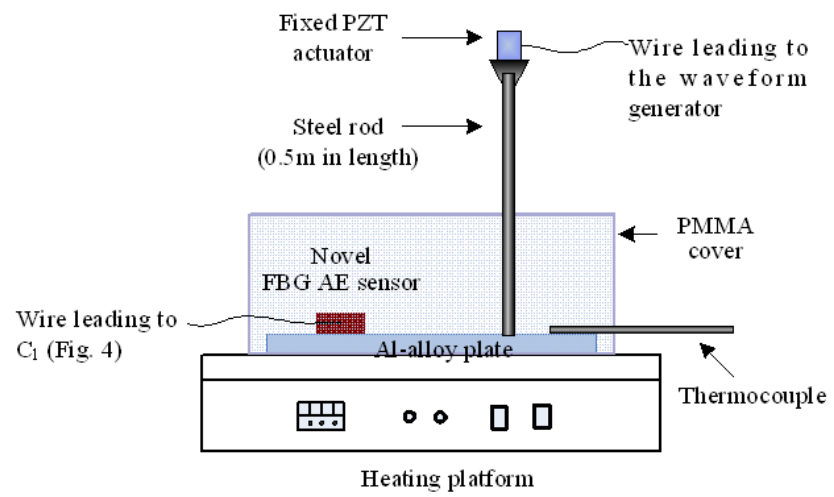

Fig. 7 Side view of the high temperature test setup.

detected signals totally have an excellent property: a high SNR. The amplitude of the frequency response keeps invariant from $25^{\circ} \mathrm{C}$ to $200{ }^{\circ} \mathrm{C}$, which will allow some variation in the $\mathrm{AE}$ source.

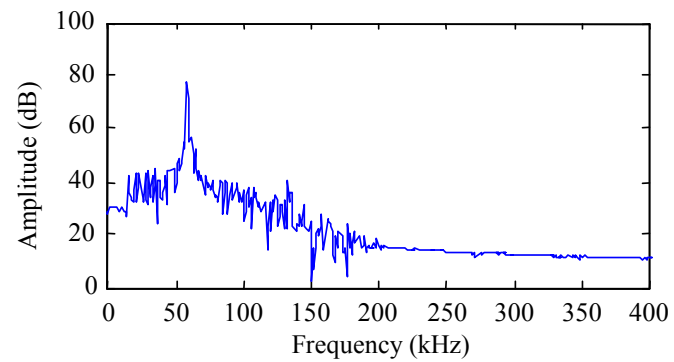

(a)

Heating platform 

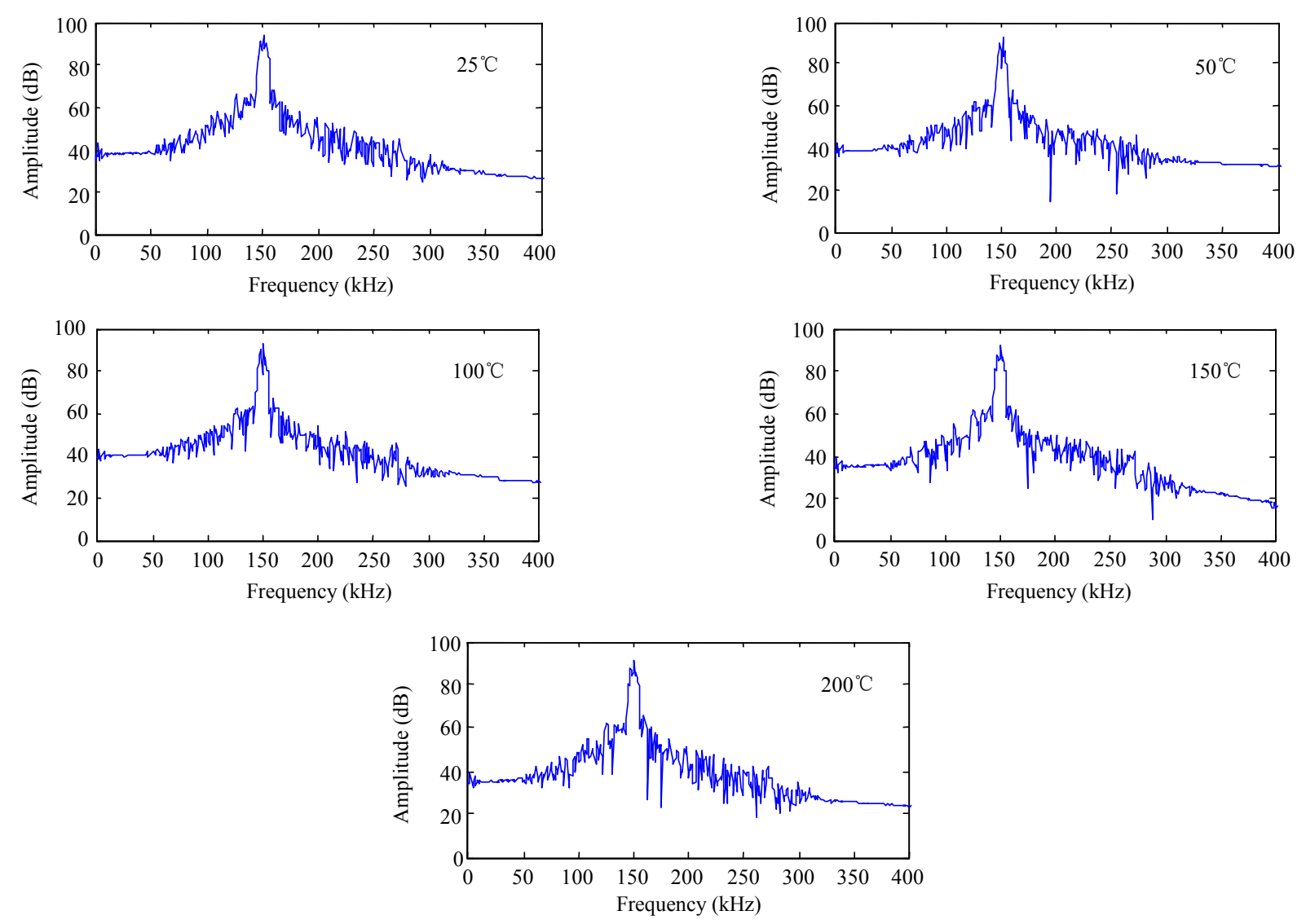

Fig. 8 Frequency response of the high temperature AE experiment.

\section{Conclusions}

In this paper, a novel high temperature FBG AE sensor is designed with a strain-insensitive configuration, a good signal noise rate can be achieved by changing the sensing length, and a high speed FBG AE detection system is also proposed based on the tunable narrow-band laser demodulation technique. The sensor resonant frequency and bandwidth measurement with respect to the sensing lengths are analyzed. Both experimental and theoretical results demonstrate that there is a clear relationship between the output response and the sensing length. Finally, the proposed FBG AE sensing system is verified by the excellent results in the high-temperature $\mathrm{AE}$ detection experiments from the ambient temperature to $200^{\circ} \mathrm{C}$. According to these results, we can expect that the designed FBG AE sensing system can be useful for the applications of AE monitoring areas at the high temperature.

\section{Acknowledgment}

This research is supported by the National Natural Science Foundation of China (Grant Nos. 61403233, 61503218, 61573226, and 61473176), the Excellent Young and Middle-Aged Scientist Award Grant of Shandong Province of China (No. BS2013DX018), and the Natural Science Foundation of Shandong Province for Outstanding Young Talents (No. ZR2015JL021).

Open Access This article is distributed under the terms of the Creative Commons Attribution 4.0 International License (http://creativecommons.org/ licenses/by/4.0/), which permits unrestricted use, distribution, and reproduction in any medium, provided you give appropriate credit to the original author(s) and the source, provide a link to the Creative Commons license, and indicate if changes were made. 


\section{References}

[1] A. Mostafapour, S. Davoodi, and M. Ghareaghaji, "Acoustic emission source location in plates using wavelet analysis and cross time frequency spectrum," Ultrasonics, 2014, 54(8): 2055-2062.

[2] Z. W. Jin, M. S. Jiang, Q. M. Sui, F. Y. Zhang, and L. Jia, "Acoustic emission source linear localization based on an ultra-short FBGs sensing system," Photonic Sensors, 2014, 4(2):152-155.

[3] T. Fu, Z. C. Zhang, Y. J. Liu, and J. S. Leng, "Development of an artificial neural network for source localization using a fiber optic acoustic emission sensor array," Structural Health Monitoring, 2015, 14(2):168-177.

[4] A. Mohimi, T. H. Gan, and W. Balachandran, "Development of high temperature ultrasonic guided wave transducer for continuous in service monitoring of steam lines using non-stoichiometric lithium niobate piezoelectric ceramic," Sensors and Actuators A: Physical, 2014, 216(1): 432-442.

[5] X. N. Jiang, K. Kim, S. J. Zhang, J. Johnson, and G. Salazar, "High-temperature piezoelectric sensing," Sensors, 2013, 14(1): 144-169.

[6] K. J. Kirk, C. W. Scheit, and N. Schmarje, "High-temperature acoustic emission tests using lithium niobate piezocomposite transducers," Insight-Non-Destructive Testing and Condition Monitoring, 2007, 49(3): 142-145.

[7] H. Zu, H. Wu, and Q. M. Wang, "High-temperature piezoelectric crystals for acoustic wave sensor applications," IEEE Transactions on Ultrasonics Ferroelectrics \& Frequency Control, 2016, 63(3): 486-505.

[8] G. H. Sun, M. Q. Bo, C. J. Zhang, and J. Z. Pan, "Propagation characteristics of acoustic signal in the circle waveguide rod of two conditions," Journal of East China University of Science and Technology (Natural Science Edition), 2010, 36(6): 851-858.

[9] N. Dixon, R. Hill, and J. Kavanagh, "Acoustic emission monitoring of slope instability: development of an active waveguide system," Proceedings of ICE: Geotechnical Engineering, 2003, 156(2): 83-95.

[10] D. S. Cheon, Y. B. Jung, E. S. Park, W. K. Song, and H. I. Jang, "Evaluation of damage level for rock slopes using acoustic emission technique with waveguides," Engineering Geology, 2011, 121(1): $75-88$.

[11] C. Crunelle, M. Wuilpart, C. Caucheteur, and P. Mégret, "Original interrogation system for quasi-distributed FBG-based temperature sensor with fast demodulation technique," Sensors and Actuators A: Physical, 2009, 150(2): 192-198.

[12] A. Minardo, A. Cusano, R. Bernini, L. Zeni, and M. Giordano, "Response of fiber Bragg gratings to longitudinal ultrasonic waves," IEEE Transactions on Ultrasonics Ferroelectrics \& Frequency Control, 2005, 52(2): 304-312.

[13] D. D. Pang and Q. M. Sui, "A relocatable resonant FBG-acoustic emission sensor with strain-insensitive structure," Optoelectronics Letters, 2014, 10(2): 96-99. 